\title{
VISUAL ANALYSIS OF DOCUMENT TRIAGE DATA
}

\author{
Zhao Geng, Robert S. Laramee \\ Visual Computing Group, Computer Science Department, Swansea University, Swansea, U.K. \\ $\{c s z g$, r.s.laramee $\} @$ swansea.ac.uk \\ Fernando Loizides, George Buchanan \\ Centre for HCI Design, City University London, London, U.K. \\ fernando.loizides.1@soi.city.ac.uk, george.buchanan.1@city.ac.uk
}

\begin{abstract}
Keywords: Information visualization, Document triage, Evaluation.
Abstract: $\quad$ As part of the information seeking process, a large amount of effort is invested in order to study and understand how information seekers search through documents such that they can assess their relevance. This search and assessment of document relevance, known as document triage, is an important information seeking process, but is not yet well understood. Human-computer interaction (HCI) and digital library scientists have undertaken a series of user studies involving information seeking, collected a large amount of data describing information seekers' behavior during document search. Next to this, we have witnessed a rapid increase in the number of off-the-shelf visualization tools which can benefit document triage study. Here we set out to utilize existing information visualization techniques and tools in order to gain a better understanding of the large amount of user-study data collected by HCI and digital library researchers. We describe the range of available tools and visualizations we use in order to increase our knowledge of document triage. Treemap, parallel coordinates, stack graph, matrix chart, as well as other visualization methods, prove to be insightful in exploring, analyzing and presenting user behavior during document triage. Our findings and visualizations are evaluated by HCI and digital library researchers studying this problem.
\end{abstract}

\section{INTRODUCTION}

Document triage is an important stage of the information seeking process. It focuses on user behavior with respect to skimming, evaluating and organizing documents when searching for information. Various studies have been conducted (Buchanan and Loizides, 2007; Loizides and Buchanan, 2009) to explore users' behavior during document triage. Over the course of these studies, a large amount of qualitative and quantitative data is collected. However, understanding and analyzing this data is difficult in its raw form. Conventionally, these experimental data are analyzed by statistical methods and simple visualizations, such as bar charts, line graphs and pie charts. These simple visualizations are useful, but of limited help for high dimensional data. Thus, there is a great demand for summarizing and presenting the data in a more insightful way that HCI scientists can better utilize. This motivates the exploitation of more advanced information visualization techniques. In recent years, we have witnessed a rapid increase in the number of visualization tools for general use, such as XMDV (Ward, 1994), Mondrian (Theus, 2002), TopCat (Mark Taylor, 2005) and ManyEyes (Viegas et al., 2007). We carry out an investigation on how well data collected by HCI and digital library researchers can be visualized by existing off-the-shelf information visualization tools and how well each can be applied. Results show that the amount of time spent on documents, pages and document features as depicted by some of our visualizations, such as the treemap, parallel coordinates, stack graph and matrix chart can help HCI and digital library scientists understand and explore user behaviors during document triage. On the other hand, we also learn that some of the visualizations, such as the 2D bar chart, 2D and 3D scatterplot are limited in their applicability to this problem. The advantages and disadvantages of the most promising visualization techniques are compared and evaluated.

In this paper we contribute the following:

- A novel attempt to systematically visualize experimental document triage data studying human be- 
haviors using state-of-the-art information visualization methods.

- We survey the variety of state-of-the-art, off-theshelf information visualization tools in order to help researchers from another domain gain insight into their experimental data.

- We compare and evaluate the various tools and visualizations with respect to their effectiveness in solving a given problem.

- The results of our investigation are evaluated by HCI and digital library researchers studying document triage.

The result of our study also provides the reader with a concise introduction to free, off-the-shelf information visualization applications and their features.

The rest of this paper is organized as follows. In section 3 we briefly review the past and related work in the study of document triage. In section 4 , we describe in detail the data collected during the document triage study. In section 6 , we investigate different visualization techniques from various tools and evaluate the usefulness of each. Section 7 presents a summary on the interactivity and scalability of visualization softwares. Section 8 contains feedback from the domain experts studying this problem. Section 9 wraps up with conclusions and the specifications for the visualization tools for HCI researchers.

\section{EXPLORATORY SPECIFICATIONS}

The data provided by the HCI group, as discussed in Section 4 is quantitative in nature, including timings, numerical ratings from participants. The main aim of a visualization to the HCI researchers is a fast overview of the data in order to formulate hypotheses on a) relationships between document properties, times and ratings and b) common recurring patterns over all the three areas mentioned in part (a). These can then be tested empirically for validity by statistical significance. Thus far, hypotheses are inferred before the study by previous results or by observing the individual behavior of participants as they perform a specific task. Indeed, many hypotheses are speculative and are sometimes based on curiosity rather than evidence. We hope that visualizations will greatly decrease the time taken to formulate more grounded hypotheses and dismiss non substantive data patterns. Furthermore, we hope to be able to test for patterns and relationships which may have previously gone unnoticed without visualization of the information.

\section{RELATED WORK}

Document triage is a highly manual process, ultimately leading to a relevance decision from the user. It is unlike the automatic information retrieval process where the decision is made by the search engine. The document triage process begins after the automatic information retrieval process and before indepth reading. The usual starting point of the document triage process is a results list. Currently, there is limited further assistance for the information seekers after the information retrieval process. A typical results list includes a title and a small description of the contents of the document (whether that is a webpage or an academic paper). In general, document triage is a fast process. From previous research we see that when presented with a results list, information seekers will make fast decisions on the relevance of documents to their information need rather than scrutinizing the full documents further (Buchanan and Loizides, 2007). Numerous previous studies have deciphered document triage behavior of information seekers (Loizides and Buchanan, 2009; Cool et al., 1993). Recent work has been geared toward summarization of documents for relevance overview. One specific area that has been gaining attention is that of tag clouds for document summarization(Gottron, 2009; Bateman et al., 2008).

Up to now, various visual analysis tools on document triage have been developed(Jonker et al., 2005; Bae et al., 2008). But these tools do not help HCI researchers visualize their user-study data. Because of the experimental nature of the study, we find it necessary to introduce more advanced existing information visualization tools to the HCI community, and help them better present and explore their various data sets.

There are many general purpose information visualization tools developed for industry, such as Eureka, SpotFire and InfoZoom (Kobsa, 2001). They provide various interactions for users to enable the "Visual Information Seeking Mantra" : overview first, zoom and filter, details on demand (Keim., 2002; Shneiderman, 1996). Advanced tools such as OpenViz (Advanced Visual Systems Inc., 2009) and ILog Discovery (Baudel, 2004), are integrated with multiple visualization techniques to handle complex data sets and queries. These tools are for commercial use. Our study focuses on free, off-the-shelf visualization software. As stated by Kobsa (Kobsa, 2001), when solving a specific problem, users, especially from other domains, might have great difficulties in selecting the most effective visualizations out of numerous choices. Also, the task questions posed from the domain experts often affect how they derive information from 
a visualization (Ziemkiewicz and Kosara, 2008). It is important to note that although there have been several general user-study evaluations of information visualizations (Kobsa, 2001; Ziemkiewicz and Kosara, 2008). This work presented here is not a general userstudy but a very specialized investigation for a focused audience, namely, document triage researchers. Although we do believe the work conducted here can benefit other users as well. Our work is to facilitate document triage experts search for visualizations that benefit most for their experimental data.

\section{BACKGROUND: USER-STUDY DATA}

The data sets used in our visualizations, is collected by our collaborated HCI researchers Buchanan and Loizides from their document triage userstudy (Loizides and Buchanan, 2009). Their experiment aims to investigate the human behaviors in the process of reading the documents, searching for information and evaluating the document relevance. During the study, 20 participants performed document triage on a closed corpus of electronic PDF documents, evaluating each for its suitability for two tasks. Log data of their interactions is captured. The documents provided for search range from short papers (2 pages) to full journal papers ( 29 pages). There are 6 documents including TABLET to TABLET5 in Task 1 , and 10 documents including HCI to HCI9 in Task 2. In Task 1, the goal is to find material on the interfaces of tablet PC's. In Task 2, the goal is to find papers on specific CHI evaluation methods. The participants' ages range from 21 to 28 . They are studying at postgraduate level in computer science discipline, and all have experience with PDF reader software. In this section we briefly describe the data collected during the study.

1. Pre-study questionnaire: Study participants filled out a questionnaire before the experiments indicating their: (1) age, (2) number of years electronic document readers have been used, (3) average number of academic documents triaged per day, (4) average amount of time per day spent searching documents. Participants were also asked to rate the importance of the following document attributes in a range from 1 to 10 ( 1 meaning "very irrelevant" and 10 meaning "very relevant"): main title, headings, introduction, plain text, conclusion, references, images and figures, highlighted and emphasized text. Participants also indicated their preference for searching on paper versus that of using a computer.

2. Data recorded for each participant during the study:
Table 1: Abbreviation of document features. Plain text means a page contains none of features from 1 to 9 . Emphasized text includes bullet point, bold text, italic text and underlined text. The abstract, keywords and general terms are features here, but not included in previous literature (Loizides and Buchanan, 2009).

\begin{tabular}{lcc}
\hline \hline Number & Features & Abbreviation \\
\hline 1 & Heading & $\mathrm{He}$ \\
2 & Abstract & $\mathrm{Ab}$ \\
3 & Keywords & $\mathrm{Kw}$ \\
4 & General Term & $\mathrm{Gt}$ \\
5 & Emphasized Text & $\mathrm{Em}$ \\
6 & Figure & $\mathrm{Fi}$ \\
7 & Conclusion & $\mathrm{Co}$ \\
8 & Reference & $\mathrm{Re}$ \\
9 & Picture & $\mathrm{Pi}$ \\
10 & Plain Text & $\mathrm{Pl}$
\end{tabular}

For each participant the total amount of time in $1 / 5$ th of a second accuracy viewing each page of each electronic document was recorded. From this the total time viewing each document can be calculated. There are 10 types of document features appearing in the study. We abbreviate the document features in order to optimize the space for visualizations, as shown in Table 1. During the study, participants' viewing time on pages is logged. Although eye-trackers are not used in the current document triage study, the HCI researchers still want to see how document features would potentially influence participants' reading patterns. Thus the viewing time on document features is inferred based on HCI researchers' hypotheses that more time spent on a page suggests more interest on the features in that page. The new findings or hypotheses obtained then will be used for further experiment design aided by the eye-trackers. The frequency of document feature on each page can be defined as $F_{i, k, q}=\frac{n_{i, k, q}}{N_{k, q}}$, where $n_{i, k, q}$ represents the number of feature $i$ appeared in page $k$ of document $q$, and $N_{k, q}$ represents the total number of all features appeared in page $k$ of document $q$.

Thus the viewing time on each feature can be estimated as $t_{i, k, q}=T_{k, q} \times F_{i, k, q}$, where $T_{k, q}$ represents the participants' average viewing time on page $k$ of document $q$.

3. Participant rating of document relevance: After each search task participants were asked to assign each document a relevance score (1 meaning "worst" or very irrelevant and 10 meaning "best" or most relevant). Also, for each document, the significance of the following document features is recorded: (1) headings (2) picture (3) figures (4) emphasized text. 


\section{OBJECTIVE RELEVANCE METRICS}

As part of this investigation we attempt to derive some objective document relevance metrics based on key terms' $T F \times I D F$ score in the document (Lee et al., 1997). These objective metrics may then be used to gain insight into how effective participants are in their search for relevant information and can also be compared with subjective metrics. The comparison of objective and subjective document relevance metrics is shown in Figure 1 and Figure 2. The details can be found in the supplementary PDF file (due to space limitations).

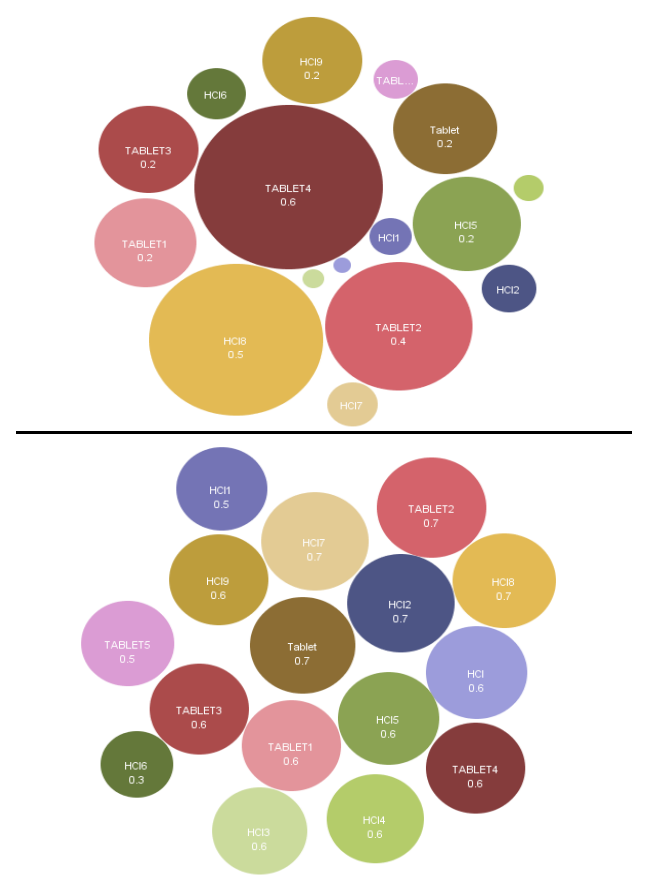

Figure 1: We can plot the subjective and objective ratings described in Section 4 and 5 onto the bubble chart in ManyEyes (Viegas et al., 2007). As shown in the figure, the top one presents the objective ratings and the bottom one shows the subjective ratings. From the bottom bubble chart, we can observe the difference of scores between documents is very slight. These two bubble charts highlight the discrepancies between the objective and subjective relevance metrics.

\section{VISUALIZATION}

In this section, we utilize various existing visualization techniques and tools to investigate document triage data. The following list summarizes the tools and their visualizations we have experimented:

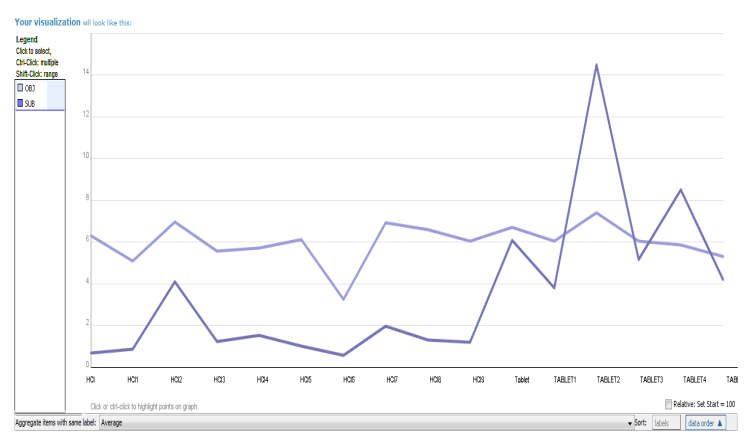

Figure 2: This figure shows the line graph plot of the subjective and objective rating scores. Documents in the order from HCI to HCI9, TABLET to TABLET6 are mapped to the x-axis. We can observe that, except documents HCI1, HCI7, HCI8 and TABLET4, the subjective rating (the line above) and the objective score (the line below) correspond in a linear fashion.

- The ManyEyes (Viegas et al., 2007) application with the following visualizations: Wordle, Tag Cloud, TreeMaps, Line Graph, Stack Graph, Bar Chart, Bubble Chart, Scatterplot, and Matrix Chart

- The XMDV (Ward, 1994) application with the following: Parallel Coordinates, Scatterplot Matrix, Star Glyphs and Dimensional Stacking

- The Mondrian (Theus, 2002) application with the following visualizations: Bar Charts, Histograms, Parallel Coordinates, Boxplots, Scatterplot Matrix

- The Treemap Application 4.1 (Kobsa, 2004): TreeMaps

- The Topcat Application (Mark Taylor, 2005): 3D Scatterplots, Histogram, Sky, Lines and Density

- Microsoft Office 2007 (Microsoft, 2007): 3D Barchart, Radar chart, 3D line graph, 3D Bubble Chart. Although Excel is a commercial application, we include it as an exception because we have a university license for this product.

- The Tableau Application (Heer et al., 2008): The free trial version only contains a few basic visualizations. Advanced options such as parallel coordinates are not available for use in the free trial.

In the following subsections, we describe the applications we used along with the visualizations of document triage data and evaluate the usefulness of each. We tried over 17 visualizations with several different variations for a total of 110 images. Each tool was systematically applied to the same data described in Section 4 (Loizides and Buchanan, 2009) by visualization researchers. For each tool, we (1) re-formated the data to match the application's input requirements, (2) tried out each of the visualiza- 


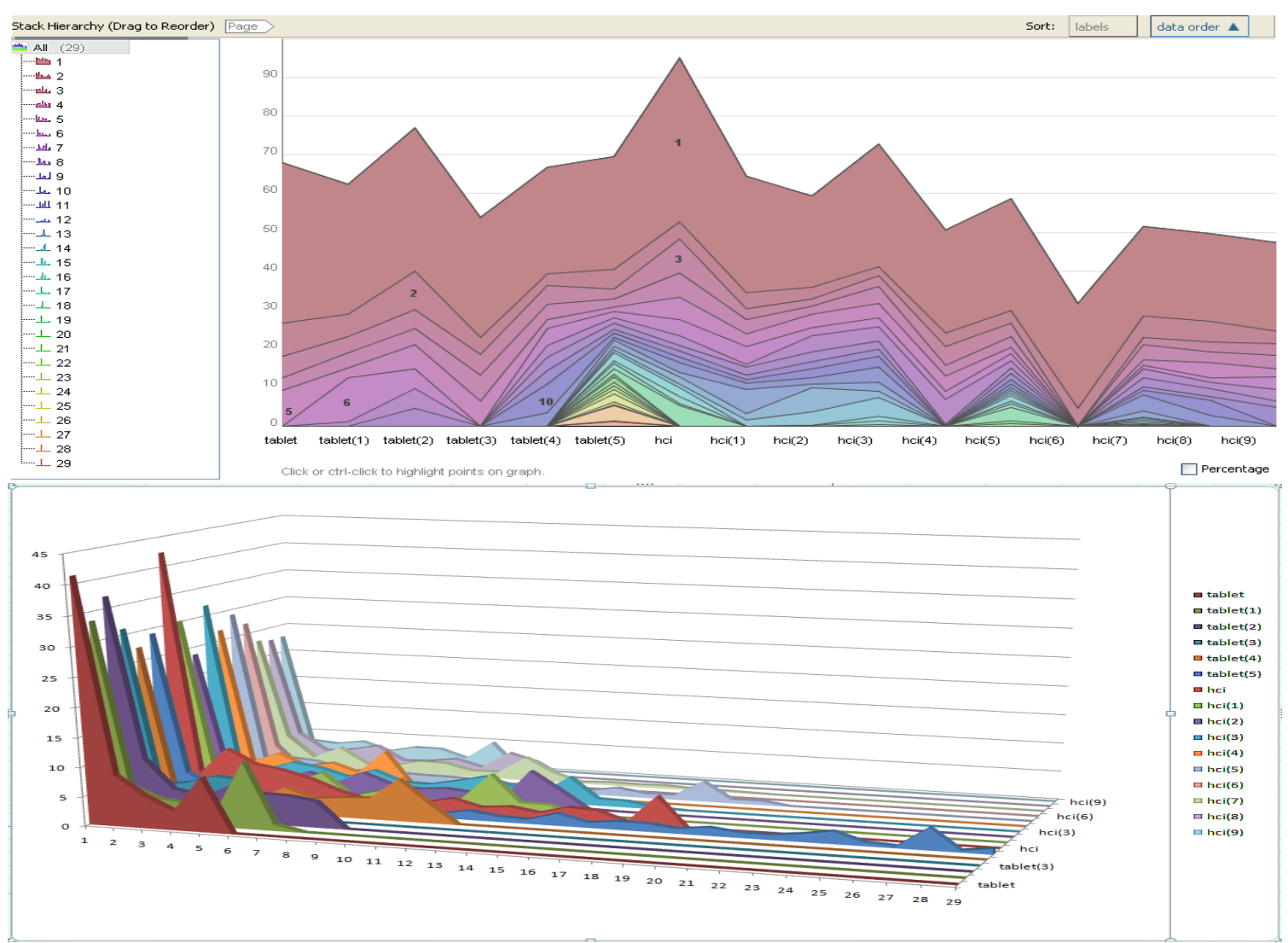

Figure 3: The top image shows the 2D stack graph visualization of document triage data in ManyEyes (Viegas et al., 2007). The X-axis represents documents in both Task 1 and Task 2. Y-axis represents the average viewing time on each page over all participants in each document. The strips in different colors represent viewing time trend for individual pages. The number in every strip indicates the page number. The bottom image illustrates the 3D stack graph plotted on Microsoft Office Excel 2007. The $\mathrm{X}$-axis is mapped to the page number, Y-axis to the viewing time and Z-axis to the documents. Compared with $2 \mathrm{D}$ stack graph, we can gain an overview of all documents' time and page distribution and compare them more intuitively.

tions offered by the tools, and (3) evaluated the utility based on the domain expert's feedback. The visualizations are assessed by domain experts - the HCI scientists who carried out the user-study (Loizides and Buchanan, 2009). Due to space limitations, we cannot describe every visualization we tried out, but only those most relevant and beneficial to the investigation. The beneficial visualizations are able to provide more insight of the data set and help the HCI researchers obtain and form new findings and hypotheses. We also provide some of the less insightful visualizations as supplementary material. Some of the less beneficial visualizations include: bar charts, bubble charts, 2D and 3D scatterplots. We provide a tour through all of the visualizations in a supplementary video. Each image of visualization, plus the supplementary video and PDF file, are stored in its original resolution on the supplementary website http//cs.swan.ac.uk/ cszg/docTriage.

\subsection{Stack Graph Visualization}

The stack graph in ManyEyes is used to visualize the total change of a group of quantities over time(Viegas et al., 2007). During the document triage study, HCI scientists observe that a user's triage process can proceed in a linear fashion starting with the first document and then reading and scoring every subsequent document (Buchanan and Loizides, 2007). The sequence of documents in Task 1 and 2 can be mapped to the time parameter of the stack graph. For each document, we can observe the changes in viewing time spent on individual pages from top image in Figure 3. This visualization shows participants spent most of their time viewing page one. Also, users spent less time on pages near the end of the documents. From the peak of each document, we can rank the documents by viewing time, e.g. HCI receives the most time and $\mathrm{HCI}(6)$ the least. Furthermore, we can compare individual pages of different documents, su- 


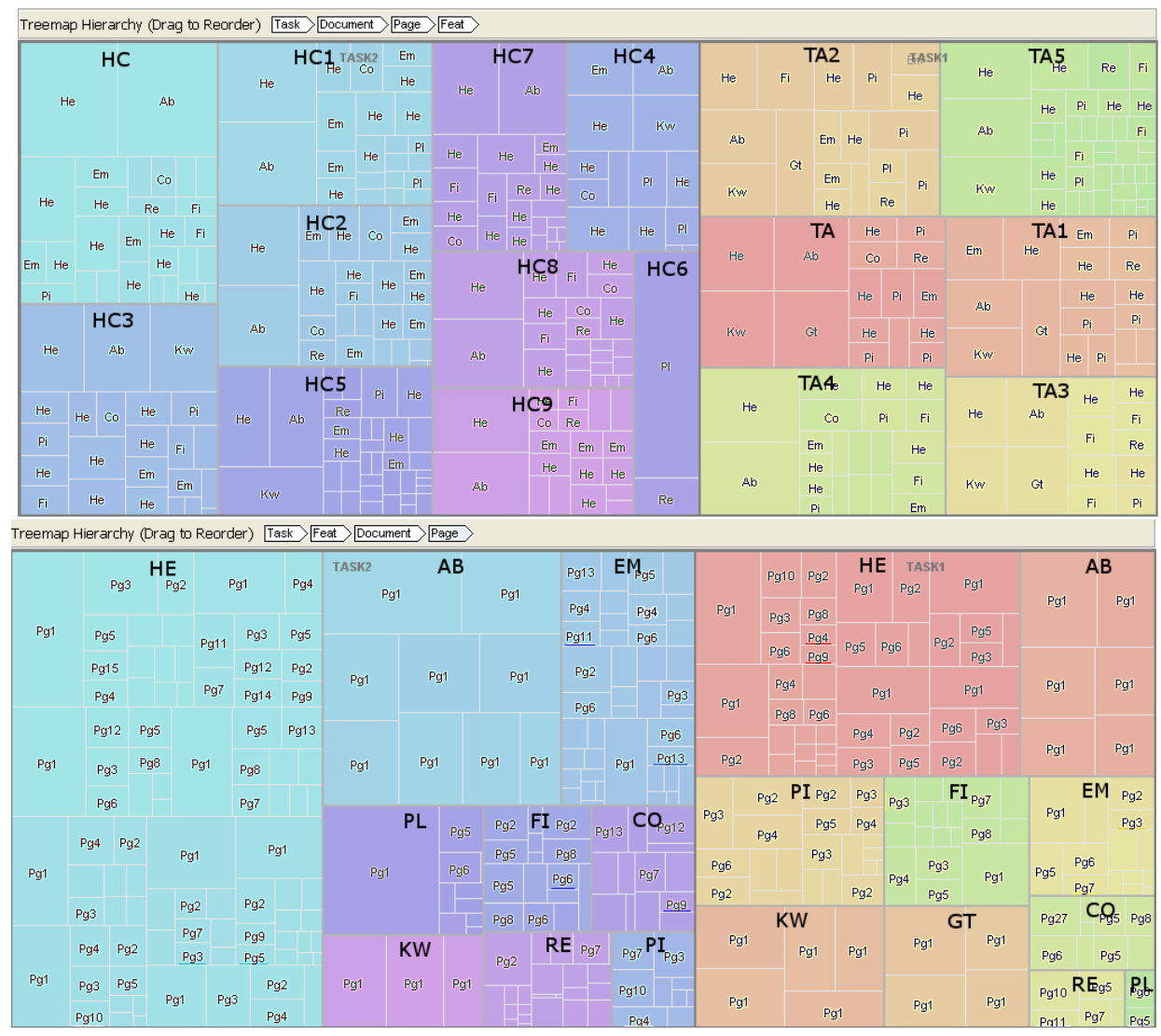

Figure 4: This figure shows the treemaps from ManyEyes (Viegas et al., 2007). The top image shows a Task-DocumentPage-Feature hierarchy. The top row of the visualization shows the current tree hierarchy. Each document name in black bold character is manually annotated. Different colors represent different documents. The document features on each page are mapped to the leaves of the tree. The bottom image shows a Task-Feature-Document-Page structure. Different colors represent distinct document features in both tasks. The feature names are manually annotated. The pages that features appear on are visualized as leaves of the structure.

ch as all pages of TABLET(2) receive more viewing time than adjacent documents.

The 2D stack graph utilizes the accurate graphical perception encodings (Cleveland and McGill, 1985), such as position, length, area, angle slope and color, to convey multiple data attributes to the user simultaneously. Also, an additional variate, namely, documents, can be included in the visualization as opposed to just two dimensions (page and time) in the bar chart, line graph or pie chart. However too many pages in this visualization leads to problems such as very thin strips or degenerate line strips. It's difficult to discern the last few pages of longer documents (10 pages more), thus the length of such documents is difficult to infer.

The problems of degenerate or overlapping strips can be reduced in the 3D stack graph, which is available in Microsoft Office Excel 2007, as shown in Figure 3 bottom. Compared with the 2D stack graph, the length of each document is clearly shown in $3 \mathrm{D}$ space. Also, we can gain a general trend of participants viewing time on documents and pages which 2D stack graph cannot offer. Although the 3D stack graph suffers from occlusion and perspective distortion (Shneiderman, 2003), with the help of the interaction techniques, such as zoom, pan, rotate and shading, the benefits provided by 3D outweigh the drawbacks in this particular case.

\subsection{Treemap}

HCI researchers study how document features influence user behaviors when searching documents. The relationship of viewing time between pages and document features may unveil user navigation patterns during document triage. In order to optimize the space to display more information, we abbreviate the nodes in the tree structure. $\mathrm{Pg} 1, \mathrm{Pg} 2$ etc. are page numbers. TA, TA1 etc. and HC, $\mathrm{HC} 1$ etc. represent documents in Task 1 and 2. The abbreviation of document featu- 


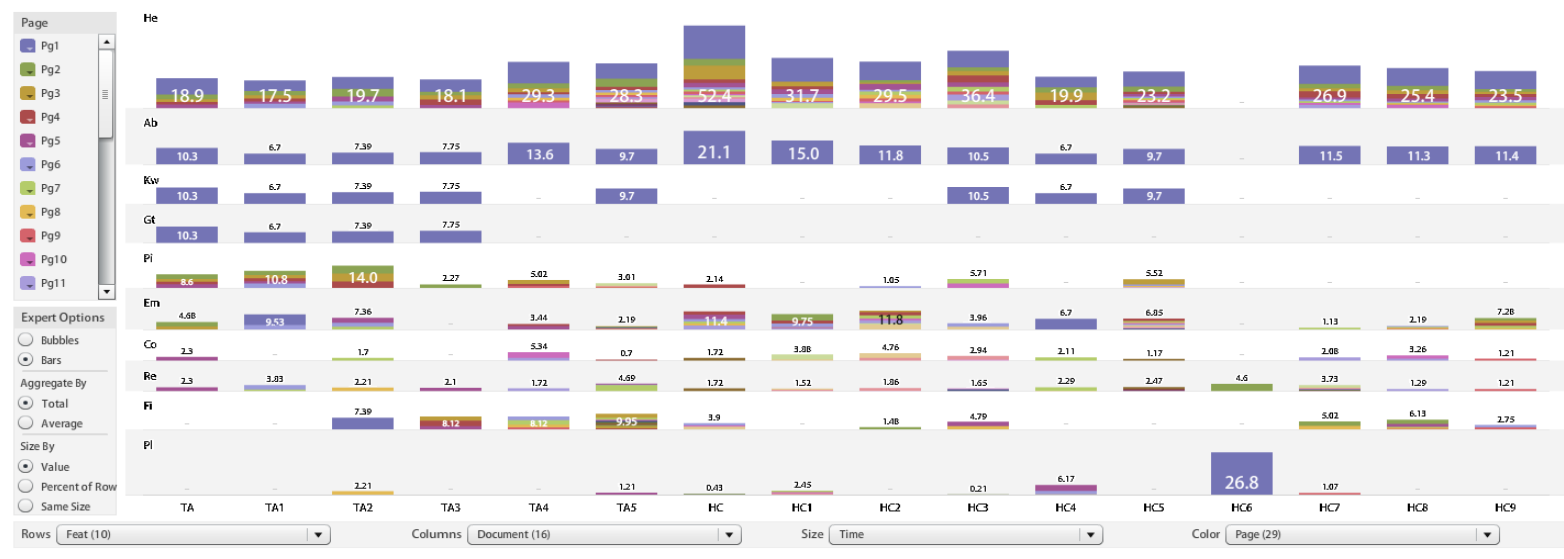

Figure 5: This image shows a matrix chart in ManyEyes (Viegas et al., 2007). Rows are mapped to the document features, columns to the documents, colors to page number and size of each bar to time. Rows, columns and colors can only accept categorical data. This visualization depicts four variates at a time and display the general view among the four variates.

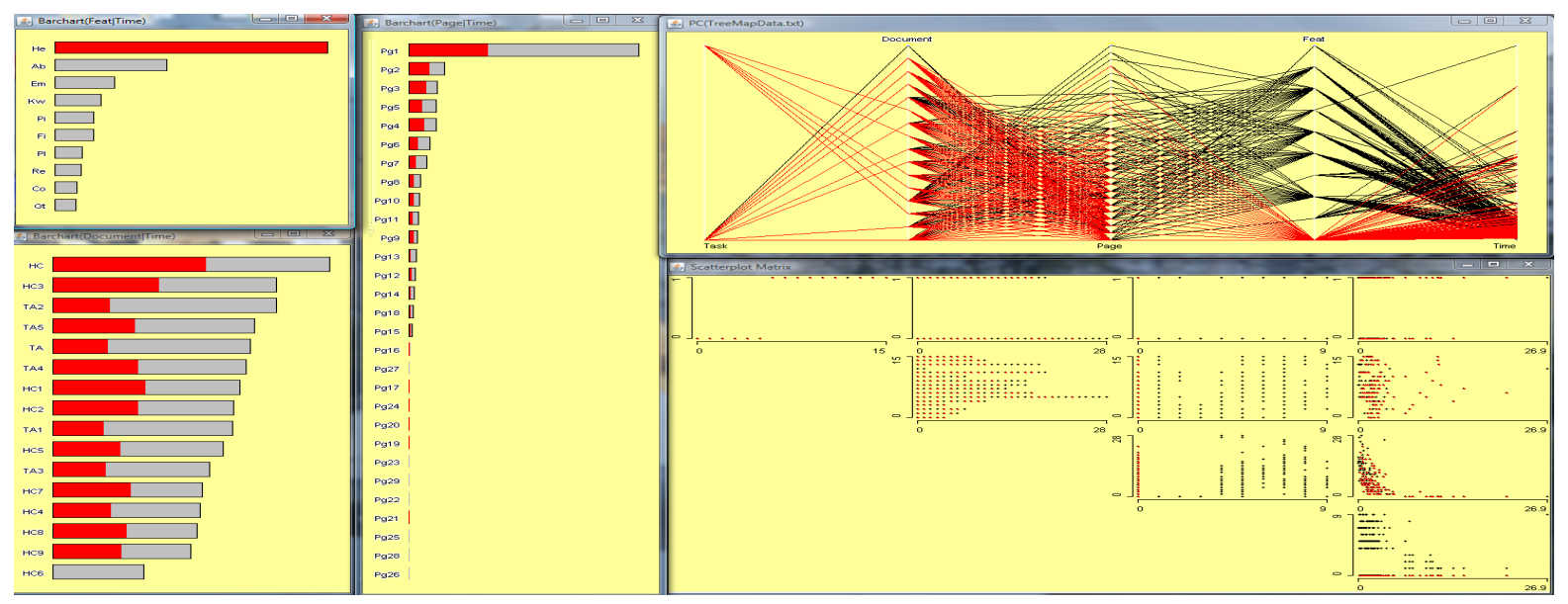

Figure 6: This figure shows a combination of bar charts, parallel coordinates and scatterplot matrix in Mondrian (Martin and Simon, 2008; Theus, 2002). There are five variates in the visualization: task, document, page number, document feature and viewing time. Plots are fully linked to one another. From these visualizations, we can observe the distribution of the highlighted feature heading $(\mathrm{He})$ in document and page respectively.

res is given in Table 1. Each page includes features, such as headings, abstract, pictures, etc. Each feature is associated with the average viewing time. The treemap is an alternative representation of tree diagram, introduced by Johnson and Shneiderman (Johnson and Shneiderman, 1991; Shneiderman, 1992). ManyEyes offers squarified treemaps, which uses rectangles with an aspect ratio close to 1 and are ordered by size (Bruls et al., 2000). It also provides various navigation such as smooth zooming, hierarchy reordering and color mapping for users to interact with different levels of the tree structure.

We can create the treemap using a TaskDocument-Page-Feature-Time hierarchy. We annotate the document names of treemap visualization result to indicate the intermediate nodes, as shown on the top in Figure 4. From this visualization, page one including its most frequent features, such as abstract $(\mathrm{Ab})$, keyword $(\mathrm{Kw})$ and headings $(\mathrm{He})$, covers the most area in all documents except "HC6". Participants almost even out the distribution of their viewing time on each document in Task 1 and on some groups of documents in Task 2, even the documents' length varies from 5 to 29 pages. We can hypothesize that participants' viewing time is mostly affected by the page one, not by the document length. With the treemap, only one level in the tree structure can be displayed each time. To compare different variates, we need to frequently switch between various tree depths, which is tedious and error-prone. Some authors try to visualize the changes of hierarchy in treemap(Tu and Shen, 2007; Blanch and Lecolinet, 2007), but such at- 
tempts are not available in the tools presented in this paper. In order to further explore participants' viewing patterns, we need a visualization which can combine documents, pages and features together in just one view. This motivates the use of matrix chart in Section 6.3

The treemap Task-Document-Page-Feature hierarchy can be switched to Task-Feature-DocumentPage order. Each task contains several distinct document features. Each feature appears in different documents. We manually annotate the document features, as shown on the bottom in Figure 4. From this visualization, we can observe the distribution of document features. Visual components, such as figures, pictures and emphasized texts, have a weaker impact than headings in terms of their population in documents and frequency in pages. Compared with Task 2 , the area in plain text (Pl) in Task 1 is dramatically reduced. This is might because featureless pages appears in 6 documents in Task 2, whereas only in 2 documents in Task 1. We also observe that pictures and figures in Task 1 cover much larger proportion than in Task 2. This is might because such features spread out in more pages in Task 1 than in Task 2. In order to further explore the influence of the feature distribution, we manually calculate participants' average viewing time on documents, and pages with figures, pictures and plain texts in both tasks. The result shows that on average, participants spent more time viewing documents in Task 1 than in Task 2. Moreover, the viewing time on pages with figures and pictures is larger than on pages only contain plain text. From these visual clues, we can form a hypotheses that pages rich in visual features, such as pictures and figures, might draw more attention than pages only containing plain text from participants. During the analysis, we need the aggregation method of numerical attributes, for example the total and average viewing time on variates appeared in the higher level than features, such as pages, documents and tasks, should be calculated and displayed. This function is not supported by the treemap in ManyEyes, but is supported in TreeMap 4.1 (Kobsa, 2004).

Compared with the stack graph, the treemap is able to co-relate four variates. It gives the researcher a clear outline of the elements in question and hierarchically classify them regarding importance.

\subsection{Matrix Chart Visualization}

Matrix chart was introduced by Marsh (Marsh, 1992). It maintains tabular organization of the data, but using bars or bubbles to represent each of the elements in the table. The matrix chart is supported in ManyEyes (Viegas et al., 2007), and variates can be mapped to four visual attributes: rows, columns, size of the bubble or bar and color, as shown on the in Figure 5 left. We adopt the bar chart for every row/column combination, because viewers can interpret changes in length more accurately than in area (Cleveland and McGill, 1985). From this visualization, we can gain an overview of four variates simultaneously. Headings $(\mathrm{He})$ are the most popular features in most documents and pages. Page one often does not contain figures and pictures. Document "HC6" jumps out as an outlier which contains the least pages and document features. We observe that document "TABLET2" is rich in visual document features, such as emphasized text (Em), pictures (Pi) and figures (Fi). Although this document only has 7 pages, it receives the second largest viewing time on average from participants. Also, we find page one in document "TABLET2" receives less viewing time than most of the initial pages on other documents. This might extend our hypotheses drawn from Section 6.2 that participants' viewing time is not only affected by the initial page, but also by the existence of visual document features in pages, such as emphasized text, pictures and figures. We also notice that pages containing a conclusion ( $\mathrm{Co}$ ) across all documents only receive little average viewing time from all participants. This seems to contradict the HCI researchers' hypotheses, which suggests that participants used to pay more attention to the document's conclusion during document triage process.

The matrix chart can present the same data set as treemap. Although it is unable to depict the hierarchies, it offers a broad view encompassing all data attributes (Marsh, 1992). Compared with treemap in Section 6.2, it provides aggregation to calculate average and total value on numerical attributes, which offers more convenience for us to explore the anomalies and patterns among four variates.

\subsection{Parallel Coordinates Visualization}

Parallel coordinates are used for displaying highdimensional data (Inselberg and Dimsdale, 1990). During the document triage study, for each participant, the percentage of his viewing time on pages with pictures, plain text, figures, conclusions and headings is calculated. The percentage of viewing time on page one and the number of conclusions each participant viewed are also recorded. This multivariate data can be plotted to seven axes on parallel coordinates in XMDV (Ward, 1994). By reordering the axes, we can find several patterns of value, as shown in Figure 7 right. There are 20 polylines in the fig- 


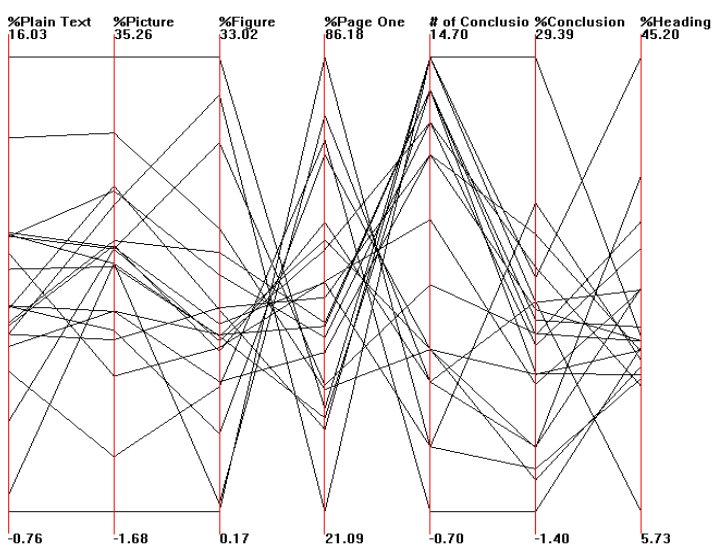

Figure 7: This image shows a parallel coordinates in XMDV (Ward, 1994). From left to right, the first four axes show the percentage of each participant's viewing time on plain text, pictures, figures and page one respectively. The last three axes show the number of conclusions each participant viewed, percentage of each participant's viewing time on conclusions and headings respectively.

ure, each one represents every participant's reading behavior on pages with document features. From this visualization, an inverse correlation between viewing time on page one and the number of viewed conclusions is clearly revealed. This implies that as participants spent more time on page one, they are likely to overlook the conclusions, and vice versa. Also, the number of conclusions being viewed and their received viewing time reveal a correlation. All the data in viewing time on figures, pictures and page one show a general trend toward inverse correlations. It could be page one often does not contain pictures and figures, as discussed in section 6.3, such that more time viewing on page one means less time is spent on figures and pictures.

Parallel coordinates are able to unveil the correlations between the viewing time of conclusions, page one, figures and pictures: observations we were unable to make with previous visualizations. A disadvantage of parallel coordinates is that large data might cause clutter which makes interpretation more difficult.

\subsection{Coordinated, Multiple Views Visualization}

Mondrian is a general purpose information visualization system. It allows multiple displays to represent one data set and links them by brushing and selection (Theus, 2002; Martin and Simon, 2008). Figure 6 shows 5 coordinated views using bar charts, parallel coordinates and scatterplot matrix, on our fivevariate data: task, document, page number, document feature and viewing time. The viewing time on documents, pages and features in the three bar charts is sorted in ascending order. Picture $(\mathrm{Pi})$ and figure $(\mathrm{Fi})$ nearly have the equal importance. The Page/Time bar chart reveals that participants focus on the first few pages, and quickly skip over the last pages. As we brush heading $(\mathrm{He})$ from parallel coordinates, the other views are updated. But the multiple views can only deal with a single table at one time. If we need to compare the participants subjective score and their estimated viewing time on the document features, we have to work in parallel with tables describing the prequestionnaire.

The power of the Mondrain is its ability to visualize arbitrary dimensions of the data set separately. Due to the limitations of screen resolution, multiple views in Mondrian may be difficult to display and interact on large data set simultaneously. Also, it can be difficult to infer which combination of visualizations is suitable and sufficient for HCI researchers to analyze their experimental data and solve the queries.

\subsection{D Bar Chart Visualization}

There are 11 distinct visualization techniques in Microsoft Excel, including various 3D visualizations for general use, and each of them has multiple variations. HCI researchers may use Excel to organize their raw data, in which arbitrary table columns can be easily mapped to the visual attributes. As shown in Figure 8 , this provides an interesting bird's-eye overview of all the participants reading behaviors on documents and pages in the experiment. From this visualization, each individual participant's reading pattern can be displayed. But it suffers from occlusion problems. This might be addressed by the user interactions, such as selection and smooth zooming, rotation, and panning. Although there's a lot of debate on 3D interface(Shneiderman, 2003; Teyseyre and Campo, 2009), considering our data set is semantically rich which contains documents, pages, participants and viewing time, we believe that the 3D bar chart is a way to further explore the individual participant's reading pattern provided that the software is able to offer enough interaction support.

\section{A BRIEF SUMMARY OF INTERACTIONS THE TOOLS}

Our goal in this paper is not a general comparison of information visualization tools, but rather a specialized comparison dedicated solely to the investigation of document triage data. The benefi- 

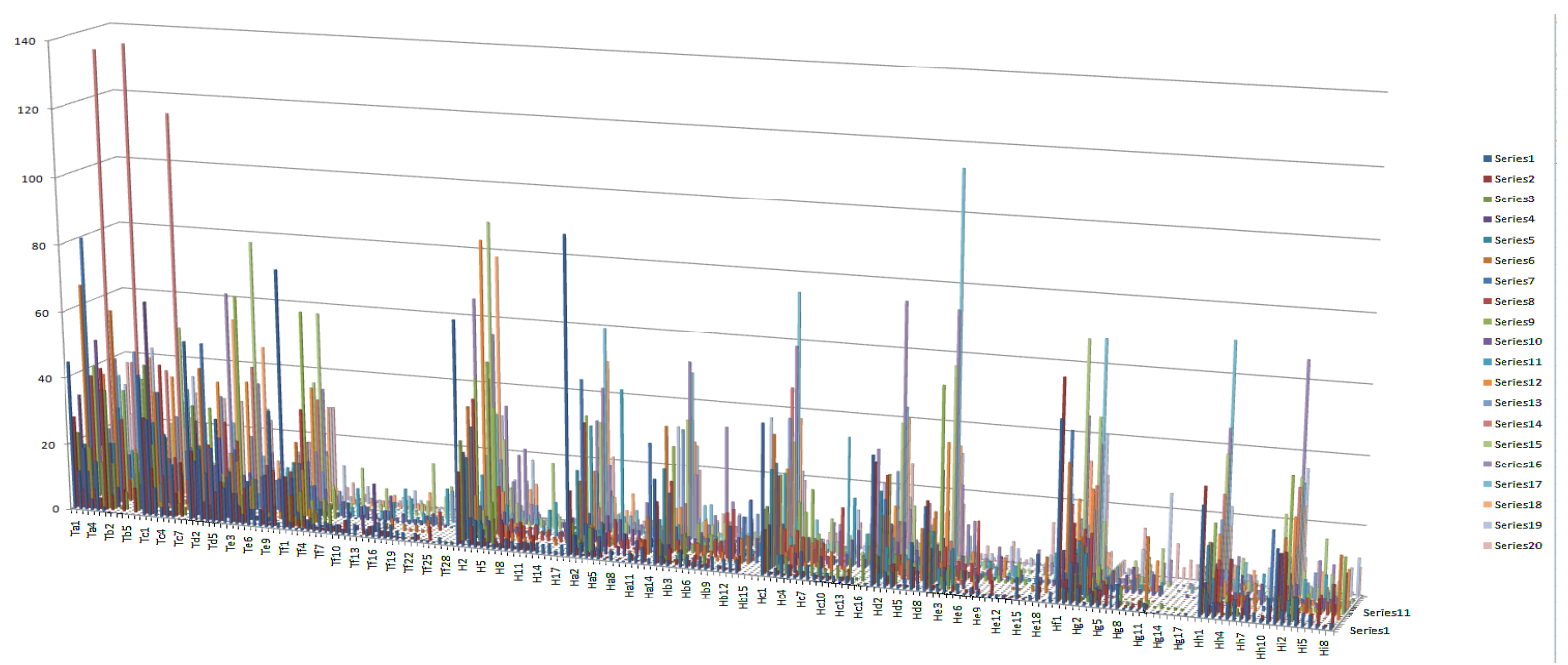

Figure 8: This figure display an overview of all 20 participants' status during the experiment in EXCEL 2007 (Microsoft, 2007). The $\mathrm{X}$-axis is mapped to the document, Y-axis to the participants and $\mathrm{Z}$-axis is the time spent on viewing documents. This visualization provides an interesting overview of the data.

\begin{tabular}{|c|c|c|c|c|c|c|c|c|c|c|c|c|}
\hline \multirow[b]{3}{*}{ Tools } & \multicolumn{6}{|c|}{ Interaction } & \multirow{2}{*}{\multicolumn{5}{|c|}{ Data Types }} & \multirow[b]{3}{*}{ 3D Vis } \\
\hline & \multicolumn{2}{|c|}{ filtering } & \multirow[b]{2}{*}{ linking } & \multirow[b]{2}{*}{ brushing } & \multirow[b]{2}{*}{ dynamic projection } & \multirow[b]{2}{*}{ dimension manipulation } & & & & & & \\
\hline & browsing & querying & & & & & $1-2 \cdot 3-2 \cdot D$ & hyper dimension & tree & text & network & \\
\hline ManyEyes & $Y$ & $\mathrm{~N}$ & $\mathrm{~N}$ & $\mathrm{~N}$ & $Y$ & $Y$ & $Y$ & $\mathrm{~N}$ & $Y$ & $\mathrm{Y}$ & $Y$ & $\mathrm{~N}$ \\
\hline XMDV & $Y$ & $Y$ & $\mathrm{~N}$ & $Y$ & $Y$ & $Y$ & $\mathrm{~N}$ & $Y$ & $\mathrm{~N}$ & $\mathrm{~N}$ & $\mathrm{~N}$ & $\mathrm{~N}$ \\
\hline Mondrian & $Y$ & $\mathrm{~N}$ & $Y$ & $Y$ & $Y$ & $Y$ & Y & Y & $\mathrm{N}$ & $\mathrm{N}$ & $\mathrm{N}$ & $\mathrm{N}$ \\
\hline TreeMap 4.1 & $Y$ & $Y$ & $\mathrm{~N}$ & $\mathrm{~N}$ & $\mathrm{~N}$ & $Y$ & $\mathrm{~N}$ & $\mathrm{~N}$ & $Y$ & $\mathrm{~N}$ & $\mathrm{~N}$ & $\mathrm{~N}$ \\
\hline TopCat & $Y$ & $Y$ & $\mathrm{~N}$ & $\mathrm{~N}$ & $\mathrm{~N}$ & $\mathrm{~N}$ & $Y$ & $\mathrm{~N}$ & $\mathrm{~N}$ & $\mathrm{~N}$ & $\mathrm{~N}$ & $Y$ \\
\hline Office 2007 & $Y$ & $\mathrm{~N}$ & $\mathrm{~N}$ & $\mathrm{~N}$ & $\mathrm{~N}$ & $\mathrm{~N}$ & $Y$ & $\mathrm{~N}$ & $\mathrm{~N}$ & $\mathrm{~N}$ & $\mathrm{~N}$ & $Y$ \\
\hline
\end{tabular}

Figure 9: For each tool, we summarize its interaction techniques and supported data types. In addition, whether a tool contains $3 \mathrm{D}$ visualizations is also recorded. In every cell of the table, "Y" denotes the specific interaction or data type is supported in that tool, "N" denotes such interaction or data type is not supported.

cial visualization tools presented in this paper are XMDV (Ward, 1994), Mondrian (Martin and Simon, 2008), ManyEyes (Viegas et al., 2007), TreeMap 4.1 (Kobsa, 2004), TopCat (Mark Taylor, 2005) and Microsoft Excel 2007 (Microsoft, 2007). They provide a variety of visualizations and integrate with different interaction options. These interaction designs of each tool are systematically applied to every visualization component within that tool. According to the taxonomy of Shneiderman (Shneiderman, 1996) and Keim (Keim., 2002), data types to be visualized can be categorized as 1-, 2-, 3-dimensional (color is mostly used to depict the third dimension in most of the tools), hyper-dimensional, text, tree and network data. In addition, based on Keim (Keim., 2002) and Kosara et al (Kosara et al., 2003)'s work and the need for visual exploration on document triage data, the most frequently used interaction techniques include filtering, brushing, linking, dimension manip- ulation, (Here the dimension manipulation includes dimension reduction and re-ordering options.) and dynamic projection. The filtering can be achieved by either a direct selection of desired subset (browsing) or by a specification of properties of the desired subsets (querying) (Keim., 2002). The dynamic projection refers to dynamically change the projection of multi-dimensional data, such as Matrix Chart in ManyEyes, and Scatterplot Matrix in XMDV and Mondrian (Ward, 1994; Theus, 2002). In this section, we present a brief summary for the tools introduced in this paper. Our summary is based on the tools' interaction designs and the scalability to various data types, as shown in Table 9.

ManyEyes is able to handle all those listed data types except for high dimensional data. During the document triage study, data gathered would usually be from an excel spreadsheet, XML document or a text file. Many-eyes provides a good precedent to 
build upon regarding raw data input for custom visualizations. Since ManyEyes is deployed on the web, it saves a lot of time for the user during software installation and configuration compared with other desktop applications. To use this application, all we need is a username and password. In terms of ease of use, the ManyEyes is no doubt the best out of the six tools to our investigation. However, because of the social and collaborative nature, the data uploaded in ManyEyes will become visible to the public. This limits its usage with respect to the data privacy.

XMDV and Mondrian, as complements to ManyEyes, are proficient in visualizing high dimensional data. XMDV features interactive, proximitybased clustering, which is effective for reducing the clutter caused by large data sets. But the structurebased bushing can be complicated to use for HCI researchers. Mondrian offers the coordinated multiple views (CMV) which effectively unveil different facets of the data. Compared with XMDV, it provides greater choice of visualizations. Not only high-dimensional data, Mondrian is quite effective in plotting large, low-dimensional data as well. Also, the input data format in Mondrian is more flexible. However, except changing the alpha value, Mondrian does not provide more advanced clutter reduction techniques, such as the clustering offered by XMDV (Ward, 1994).

The TreeMap 4.1 (Kobsa, 2004) is specifically designed to implement the treemap. Compared with ManyEyes, it offers much more interaction options, such as numerical aggregation, various layouts, filtering and etc. However, with respect to the aesthetic feel of the visualization, the HCI experts prefer ManyEyes which provides more aesthetically pleasing color coding and the animation when traversing through the different hierarchies.

Excel and TopCat are the only tools offering the $3 \mathrm{D}$ visualization through the tools presented in this paper. Although there is a lot of debate on $3 \mathrm{D}$ visualization (Shneiderman, 2003), it's surprising that the HCI researchers show more preference in 3D scatterplot and bar chart shown in Figures 8 and 3. However, in order to completely exploit the potential of $3 \mathrm{D}$ visualizations, the interaction supports are very important. Although changing the viewing perspective, such as rotation, zooming and pan, are provided in Excel and TopCat, the shading, which can effectively depict the depth information, is missing in both tools.

The first factor that became apparent is that no one visualization or tool on its own can identify all patterns and behaviors needed to be tested. Also, from the Table 9, we can see that no tool is able to sup- port all of the data types and interactions. Furthermore, some visualizations like the bubble chart can cause the researcher to miss patterns and make false inference, such as introduced in Section 8. In light of this, it would be reasonable for a bespoke tool to include several visualizations in parallel. Therefore, a coordinated multiple view application allowing for a) several visualizations of the same data and b) one visualization with different data sets is needed. Ideally, visualizations for the document triage data would include: the line graph, 3D stack graph, treemaps and parallel coordinates.

Overall, visualizations are underused in the HCI community as a means of interacting with extracted data sets. In this research we have explored the ways in which the visualizations enrich the exploration of relationships between different data sets of the same study. As an exploratory tool, using these visualizations provides insight into hypotheses formulation about our data that is not evident from raw material. It is the aim of future work to apply the visualizations presented here, as well as further visualizations to not only explore the deciphering of the raw data, but to also assist users performing triage in making inferences about their material.

\section{DOMAIN EXPERT REVIEW}

We, the domain experts, are impressed to see a multitude of visualizations that can represent our data. What became immediately evident was the ease and speed at which these visualizations could be produced. We systematically went through the visualizations identifying the immediate inferences that would have been possible before statistical analysis, but also factors that may obfuscate useful hypotheses from being formed. Analysis thus far has relied on statistical scrutiny such as t-tests. Although these are necessary for verifying a relationship or pattern they do not provide good means for exploration of the data. Here, we discuss the most significant observations and compare some of the visualizations presented in the paper.

The first visualization that caught our attention was the bubble chart in Figure 1 which compared the participants' subjective scores for the relevance of the documents with the (TFxIDF) (Lee et al., 1997) scores. By simply viewing the bubble chart visualization we are mistakenly led to think that due to the unevenness of the size of the bubbles, that there is no correlation between the occurrence of popular terms in the documents and the participant rankings. We note that this is a test that was not performed when looking at the data without the help of visualizations. 
We were surprised to then observe the line graph visualization in Figure 2 which revealed a relationship between term occurrence and document ratings. It seems that, although the sizes of the subjective bubbles in the first visualization were a different size than the objective bubbles, the size proportion between the corresponding documents in each category has a positive correlation. We can see from this that the bubble visualization would be useful to our work when comparing two groups, but care needs to be taken to match the correct type of variables for the two groups. Due to this limitation and risk of misinterpretation of the data it is deemed quite difficult to apply the bubble chart visualization to effective exploratory research in our work. We are however, convinced that the line graph visualization can produce much more accurate overviews of relations between groups and patterns.

Closely related in information representation is the treemap visualization in Figure 4. The flexibility that this visualization offers in manually changing the hierarchy of the data to be processed gives it an advantage. Furthermore, the representation areas give a much clearer means of comparing features and timings. However, as hypothesized from this visualization, it lacks specific detail. For this visualization to be successful at providing useful specific information to an $\mathrm{HCI}$ researcher would need further interaction capabilities.

Another beneficial representation of the data is found on the 3D stack graph in Figure 3. Beyond giving more information that the closely related 2D stack graph (which basically gives us the average values of all the pages) it allows us to detect further interesting behaviors worth exploring. For example, we notice the importance of the first page, but also the steady decline in attention as the page count increases. We also observe the peaks close to the ends which requires further scrutiny, but also that the decline in attention is mostly steady. The 'anomalies' in the decline attest to one of two things: a) a sharp drop in attention on a specific point in the document or b) an increased amount of attention. We can therefore infer that further features also attract attention and test for the impact each feature has on attention.

One of the most interesting visualizations we came across was that of parallel coordinates on the right in Figure 7. The features of a document and the influence they have time wise on participants constitutes a very important part of our data pool. This visualization gives us a clear image as to the percentage of time spent on those features in an-easy-to-compare format. Although there is great potential for this specific visualization, we do have two criticisms. The first is regarding the upper and lower limit of the ver- tical axis. For every feature the maximum percentage time is set to the upper limit and therefore giving a false comparison between the feature values. This should be remedied in order to facilitate clearer comparative abilities. Another improvement which would increase comparative ability between data sets would be to be able to produce superimposed average values, standard deviations and multiple side by side visualizations of data sets.

\section{CONCLUSIONS AND FUTURE WORK}

We have surveyed a range of off-the-shelf, freely available information visualization tools for the visual analysis and investigation of document triage. Although there are many options available, only a selected few visualizations are useful for this particular application. The beneficial visualizations are able to reveal the relationship and get more insight among the document triage experimental data sets, which have high dimensions and contain both categorical and numerical data types. In this paper, we have carried out the exploratory tasks on these data sets. A range of less beneficial visualizations, and the full set of all visualizations are provided via a supplementary material and video stored in our url. Our study also serves as a useful tool for readers interested in gaining an overview of existing, free, state-of-the-art information visualization tools. We also report positive and constructive feedback from experts in the HCI and digital library domain. Since summarized representations of documents rely heavily on text presented to the user. In the future, we will focus on another interesting visualization that has potential in the document triage process, namely the text visualization, such as Wordle (Viegas et al., 2009), which allows for highlighting the most frequently occurring terms hinting at the importance of the document to the information need of a seeker.

\section{REFERENCES}

Advanced Visual Systems Inc. (2009). OpenViz. 300 Fifth Avenue,Waltham, MA 02451. http://www.avs.com.

Bae, S., Hsieh, H., Kim, D., Marshall, C., Meintanis, K., Moore, M., Zacchi, A., and Shipman, F. (2008). Supporting Document Triage via Annotation-based Visualizations. In Proceedings of the American Society for Information Science and Technology, volume 45, pages 1-16.

Bateman, S., Gutwin, C., and Nacenta, M. (2008). Seeing Things in the Clouds: The Effect of Visual Features 
on Tag Cloud Selections. In Proceedings of the nineteenth ACM conference on Hypertext and hypermedia, pages 193-202, New York, NY, USA. ACM.

Baudel, T. (2004). Browsing Through an Information Visualization Design Space. In Proceedings of ACM CHI Conference on Human Factors in Computing Systems, volume 2 of Demonstrations, pages 765-766.

Blanch, R. and Lecolinet, E. (2007). Browsing Zoomable Treemaps: Structure-Aware Multi-Scale Navigation Techniques. IEEE Transactions on Visualization and Computer Graphics, 13(6):1248-1253.

Bruls, M., Huizing, K., and van Wijk, J. J. (2000). Squarified Treemaps. In Proceedings of Joint Eurographics/IEEE TVCG symposium Visualization, pages 3342.

Buchanan, G. and Loizides, F. (2007). Investigating Document Triage On Paper And Electronic Media. In Proceedings of the European Conference on Research and advanced Technology for Digital Libraries, 4675:416-427.

Cleveland, W. S. and McGill, R. (1985). Graphical Perception and Graphical Methods for Analyzing Scientific Data. Science, 229(4716):828-833.

Cool, C., Belkin, N. J., Frieder, O., and Kantor, P. (1993). Characteristics of Texts Affecting Relevance Judgments. In In 14th National Online Meeting, pages $77-84$.

Gottron, T. (2009). Document Word Clouds: Visualising Web Documents as Tag Clouds to Aid Users in Relevance Decisions. In Research and Advanced Technology for Digital Libraries, 13th European Conference, Proceedings, volume 5714 of Lecture Notes in Computer Science, pages 94-105. Springer.

Heer, J., Mackinlay, J. D., Stolte, C., and Agrawala, M. (2008). Graphical Histories for Visualization: Supporting Analysis, Communication, and Evaluation. IEEE Transactions on Visualization and Computer Graphics, 14(6):1189-1196.

Inselberg, A. and Dimsdale, B. (1990). Parallel Coordinates: A Tool for Visualizing Multi-dimensional Geometry. In Proceedings of IEEE Visualization, pages 361-378.

Johnson, B. and Shneiderman, B. (1991). Tree Maps: A Space-Filling Approach to the Visualization of Hierarchical Information Structures. In Proceedings of IEEE Visualization, pages 284-291.

Jonker, D., Wright, W., Schroh, D., Proulx, P., and Cort, B. (2005). Information Triage With Trist. In Proceedings of Intelligence Analysis, pages 1-6.

Keim., D. A. (2002). Information Visualization and Visual Data Mining. IEEE Transactions on Visualization and Computer Graphics, 8:1-8.

Kobsa, A. (2001). An Empirical Comparison of Three Commercial Information Visualization Systems. In Proceedings of IEEE Symposium on Information Visualization, San Diego, CA, pages 123-130.

Kobsa, A. (2004). User Experiments with Tree Visualization Systems. In Proceedings of IEEE Symposium on Information Visualization, pages 9-16. IEEE Computer Society.
Kosara, R., Hauser, H., and Gresh, D. (2003). An Interaction View on Information Visualization. In Proceedings of Eurographics, pages 123-137.

Lee, D. L., Chuang, H., and Seamons, K. E. (1997). Document Ranking and the Vector-Space Model. IEEE Software, 14(2):67-75.

Loizides, F. and Buchanan, G. (2009). An Empirical Study of User Navigation during Document Triage. In Proceedings of Research and Advanced Technology for Digital Libraries, 13th European Conference, volume 5714 of Lecture Notes in Computer Science, pages 138-149. Springer.

Mark Taylor (2005). TOPCAT - Tool for OPerations on Catalogues And Tables Version 3.4-3. Starlink development.

Marsh, S. (1992). The Interactive Matrix Chart. ACM SIGCHI Bulletin, 24(4):32-38.

Martin, T. and Simon, U. (2008). Interactive Graphics for Data Analysis: Principles and Examples (Computer Science and Data Analysis). Chapman \& Hall/CRC.

Microsoft (2007). Microsoft Office Excel 2007 product guide. Microsoft office.

Shneiderman, B. (1992). Tree Visualization With Treemaps: a 2-d Space-filling Approach. ACM Transactions on Graphics, 11(1):92-99.

Shneiderman, B. (1996). The Eyes Have It: A Task by Data Type Taxonomy for Information Visualizations. In Proceedings of IEEE Symposium on Visual Languages, pages 336-343.

Shneiderman, B. (2003). Why Not Make Interfaces Better than 3D Reality? IEEE Computer Graphics and Applications, 23(6):12-15.

Teyseyre, A. R. and Campo, M. R. (2009). An Overview of 3D Software Visualization. IEEE Transactions on Visualization and Computer Graphics, 15(1):87-105.

Theus, M. (2002). Interactive Data Visualization Using Mondrian. Journal of Statistical Software, 7(11):1-9.

Tu, Y. and Shen, H.-W. (2007). Visualizing Changes of Hierarchical Data using Treemaps. IEEE Transactions on Visualization and Computer Graphics, 13(6):1286-1293.

Viegas, F. B., Wattenberg, M., and Feinberg, J. (2009). Participatory Visualization with Wordle. IEEE Transactions on Visualization and Computer Graphics, 15(6):1137-1144.

Viegas, F. B., Wattenberg, M., van Ham, F., Kriss, J., and Mckeon, M. (2007). ManyEyes: A Site for Visualization at Internet Scale. IEEE Transactions on Visualization and Computer Graphics, 13(6):1121-1128.

Ward, M. O. (1994). XmdvTool: Integrating Multiple Methods for Visualizing Multivariate Data. In Proceedings of IEEE on Visualization, pages 326-336. IEEE Computer Society Press.

Ziemkiewicz, C. and Kosara, R. (2008). The Shaping of Information by Visual Metaphors. IEEE Transactions on Visualization and Computer Graphics, 14(6):1269-1276. 